\title{
Relaciones cuantitativas entre los contenidos de azufre y de sulfuros en los aceros inoxidables tipo AISI 303: influencia de la composición química de los sulfuros ${ }^{(\bullet)}$
}

\author{
J. Botella $(*)$, C. Merino $(* *)$, M.A. Heredia $(*)$, J. Almagro $^{(*)}$ \\ Resumen Se trabaja con una serie de diez redondos de aceros inoxidables tipo AISI 303 de composiciones quí- \\ micas prácticamente iguales a excepción del azufre que varía entre 0,25 y $0,36 \%$. Se examinan los \\ aceros mediante Microscopía Electrónica de Barrido (MEB) y Espectrometría de Dispersión de Ener- \\ gías de Rayos X (EDX) acoplada al MEB, analizándose químicamente los sulfuros y determinando, \\ mediante análisis automático de imágenes, los volúmenes ocupados por los sulfuros. Parece deduci- \\ ble que la composición química de los sulfuros en el AISI 303 puede ser discretamente variable aún \\ siendo prácticamente invariable la composición química del acero. Ello podría significar que las con- \\ diciones de solidificación y las transformaciones termomecánicas pueden influir, dando como resul- \\ tado variaciones en la composición química de los sulfuros. Se establecen relaciones cuantitativas \\ entre los contenidos de azufre y de sulfuros y se estudia la influencia que en dichas relaciones tienen \\ las composiciones químicas de los sulfuros. Se comparan los resultados experimentales con los obte- \\ nidos a partir de cálculos teóricos, obteniéndose un buen grado de aproximación cuando se tiene en \\ cuenta que los sulfuros reales tienen composiciones químicas distintas de la del sulfuro teórico, $\mathrm{MnS}$.
}

Palabras clave: Acero inoxidable. Azufre. Sulfuros. Microanálisis de sulfuros. Volúmenes de inclusiones tipo sulfuros.

\section{Quantitative relations between sulphur and sulphide contents in AISI 303 stainless steels: influence of the sulphide chemical composition}

\begin{abstract}
The work has been done with ten AISI 303 stainless steel rods which have similar chemical compositions, except for sulphur which varies between 0.25 and $0.36 \%$. These steels are studied by Scanning Electron Microscopy (SEM) and Energy Dispersive X-Ray Spectrometry (EDX). The sulphides are chemically microanalysed and the occupied volume determined by automatic image analysis. It can be inferred that the chemical composition of sulphides in AISI 303 steels is variable to some extent even though the steel chemistry is almost invariable. This could mean that solidification conditions and thermomechanical transformations are able to affect resulting in variations of sulphide compositions. Quantitative relations between sulphur and sulphide contents are established and the influence of sulphide chemical compositions on those relations is studied. The experimental results are compared with those obtained from theoretical calculations. A good approach is obtained when the difference between chemical compositions of real sulphides and the theoretical one, MnS, is taken into account.
\end{abstract}

Keywords: Stainless steel. Sulphur. Sulphides. Sulphide microanalysis. Volume of sulphide-type inclusions.

$(\bullet \quad$ Trabajo recibido el día 29 de octubre de 1998 y aceptado en su forma final el día 25 de mayo de 1999.

(*) ACERINOX, S.A. Centro de Investigación y Ensayos. 11379 Palmones, Los Barrios, Cádiz (España).

(**) Dpto. de Ciencia de los Materiales e Ingeniería Metalúrgica. Facultad de Ciencias Químicas. UCM. 28040 Madrid (España).

\section{INTRODUCCIÓN}

El análisis químico del acero permite conocer su contenido de azufre, de modo que si se conociera la solubilidad de este elemento en el acero sólido y la composición química y estructural de los sulfuros, 
se podría calcular fácilmente la cantidad de sulfuros a formar a partir de un cierto contenido de azufre insoluble. Pero la solubilidad del azufre en los aceros no está bien establecida y depende, entre otros factores, de la composición química y de la estructura cristalina del acero. Pero a la vez, la composición química y estructural de los sulfuros depende tanto de la del acero (contenidos de azufre, manganeso, cromo, etc.) como de los equilibrios metalúrgicos durante los procesos de acería. Todo lo anterior se puede traducir en que, aunque se conozca la cantidad de azufre de un acero, no parece tan sencillo deducir ni la cantidad ni el volumen de los sulfuros presentes en dicho acero.

Los inoxidables AISI 303 son clásicos $18 \mathrm{Cr} 8 \mathrm{Ni}$, en los que la aleación con cantidades relativamente reducidas de azufre (entre 0,2 y $0,4 \%$ en masa) produce la formación de inclusiones tipo MnS en cantidades, formas y distribuciones tales que facilitan extraordinariamente los procesos de mecanización que implican arranque de virutas. En estos aceros en los que, junto al carácter de inoxidables prima la facilidad de mecanización y ésta se deriva de la cantidad y calidad de los sulfuros formados, es importante conocer con profundidad todo aquello que relacione una medida directa e inmediata (análisis químico de azufre en el acero) con aquellas otras características (cantidades y composiciones químicas de los sulfuros) de las que depende el adecuado funcionamiento para el que dichos aceros fueron diseñados y fabricados.

El desconocimiento de la solubilidad del azufre en los inoxidables es una dificultad ya comentada en el planteamiento de la problemática. Los autores que tratan el tema ( 1 y 2), señalan que la solubilidad del azufre en el acero es muy baja y que todo el azufre del acero está prácticamente en forma de sulfuros. Fujiwara et al. (3), dan cifras de 9 y 20 ppm de azufre soluble en aceros $18 \mathrm{Cr} 8 \mathrm{Ni}$ con contenidos de manganeso del 1 y del $0,2 \%$ en masa respectivamente. Otros trabajos (4), encuentran $11 \mathrm{ppm}$ como solubilidad del azufre en aceros $18 \mathrm{Cr} 8 \mathrm{Ni1}, 5 \mathrm{Mn}$, dato en franca coincidencia con los de Fujiwara et al. (3).

El tipo de acero AISI $304(18 \mathrm{Cr} 8 \mathrm{Ni})$ limita el contenido de azufre a menos de $0,030 \%$ en masa y los contenidos típicos de azufre en los aceros comerciales son inferiores al $0,010 \%$. Suponiendo que el acero tuviese un contenido de $\mathrm{S}=0,005 \%$ y aceptando, de acuerdo con los autores (3 y 4), un dato de solubilidad de azufre del $0,001 \%$, podríamos decir que hasta un $20 \%$ de todo el azufre del AISI 304 no estaría implicado en la formación de sulfuros. Consecuentemente, la solubilidad del azufre es un dato que puede ser relevante en el AISI 304 , en la medida que sea un objetivo el evitar absolutamente la presencia de inclusiones de sulfuros (3), o que lo sea el estudio de los volúmenes de sulfuros formados a partir de una cierta cantidad de azufre en el acero (4).
Sin embargo, en el caso del AISI 303, en el que el azufre es un aleante formador de sulfuros, solubilidades del $0,001 \%$ son prácticamente despreciables frente a las concentraciones habituales de azufre en estos aceros $(0,2-0,4 \%)$. En consecuencia y para el AISI 303 sí puede decirse que, de hecho, todo el azufre de aleación va a estar formando sulfuros y que la solubilidad del azufre en el acero no afectará, en la práctica, a la relación que pueda establecerse entre el porcentaje en masa de azufre en el acero y el porcentaje en volumen de sulfuros en dicho acero.

Las composiciones químicas de los sulfuros del inoxidable dependen del contenido en manganeso del acero (5 y 6), de modo que se puede escribir la fórmula genérica MnxCrySz. Para bajos contenidos de manganeso se formarán sulfuros mayoritariamente de cromo, mientras que para contenidos relativamente altos de manganeso los sulfuros serán ricos en manganeso.

El contenido típico de manganeso en el acero AISTI 303 es del 1,8\%, de modo que asegurando la relación (\% masa $\mathrm{Mn}):(\%$ masa $\mathrm{S})=6$, es decir $1,8: 0,3=6$, se asegura que los sulfuros formados serán del tipo MnS, que facilitarán la capacidad de mecanización del acero. Así pues, asumiendo la práctica insolubilidad del azufre en el acero y que los sulfuros formados fuesen precisamente $\mathrm{MnS}$ y que la densidad (7) de dichos sulfuros fuese de 4,03 $\mathrm{g} \cdot \mathrm{cm}^{-3}$, podría plantearse un cálculo correlacionando el porcentaje en masa del azufre del acero y el porcentaje en volumen de los sulfuros presentes. Este trabajo supone una aportación en este sentido y plantea los siguientes objetivos:

- la determinación experimental de los volúmenes y de las composiciones químicas de los sulfuros en típicos AISI 303 con cantidades variables de azufre.

- el estudio de las relaciones existentes entre los volúmenes y composiciones químicas de los sulfuros y los contenidos de azufre de los aceros, a partir de los datos experimentales.

- las deducciones teóricas de las relaciones existentes entre los contenidos de azufre de un acero y los volúmenes de sulfuros que se pueden formar en tal acero, en función de la composición química de los mismos.

- la comparación de las relaciones obtenidas a partir de los datos experimentales y de las deducciones teóricas.

\section{MATERIALES Y MÉTODOS EXPERIMENTALES}

Se parte de diez palanquillas de $145 \mathrm{~mm} \times 145 \mathrm{~mm}$ de AISI 303 procedentes de coladas en continuo, con 
composiciones químicas prácticamente iguales a excepción del azufre, elemento en estudio. Las palanquillas se laminan en caliente a redondos de 6 a 10 mm de diámetro. Finalmente se recuecen y decapan. Los materiales se analizan químicamente mediante espectrometría de fluorescencia de rayos $\mathrm{X}$ y analizadores de carbono, azufre, nitrógeno y oxígeno.

Se preparan secciones metalográficas transversales a los ejes longitudinales de los redondos. Para el examen inclusionario se emplea un microscopio electrónico de barrido HITACHI S-570 que cuenta con una pletina cuyos ejes X e Y se encuentran motorizados (motorización SESAME, de KEVEX). El sistema de microanálisis por dispersión de energías de rayos $\mathrm{X}$ acoplado al MEB es un KEVEX 8005 , dotado con un digitalizador y analizador de imágenes de electrones y de rayos X.

$\mathrm{El}$ análisis inclusionario contempla dos etapas: análisis de la forma y ocupación de los sulfuros y análisis químico de los mismos.

El sistema de imágenes se ha calibrado con un patrón de cristal de silicio con cuadrados de $10 \cdot 10 \mu \mathrm{m} \pm 0,1 \mu \mathrm{m}$ (8). La calibración se realiza para las condiciones normalizadas de trabajo, que se encuentran recogidas en la tabla I.

Se elige la señal de electrones retrodispersos para las imágenes a analizar ya que permite diferenciar claramente los sulfuros de la matriz metálica y de las otras inclusiones del material, debido a la gran diferencia en los números atómicos promedio.

Las imágenes de electrones retrodispersos a digitalizar se han seleccionado de acuerdo con la figura 1 (24 imágenes por sección), examinando el estado inclusionario del material en el núcleo del redondo (es decir, la zona alrededor del centro), las zonas intermedias y las zonas próximas a la periferia (9-11).

Llamando $\mathrm{R}$ al radio del redondo, las áreas más externas se seleccionan a una distancia R/40 de la superficie, las del núcleo se toman a una distancia

TABLA I.- Condiciones de obtención de imagen

TABLE I.-Conditions for image acquisition

\begin{tabular}{|l|l|}
\hline Tipo de señal: & $\begin{array}{l}\text { Electrones } \\
\text { retrodispersos }\end{array}$ \\
$\begin{array}{l}\text { Potencial de } \\
\text { aceleración del haz } \\
\text { de electrones }\end{array}$ & $25 \mathrm{keV}$ \\
$\begin{array}{l}\text { Distancia } \\
\text { de trabajo }\end{array}$ & $15 \mathrm{~mm}$ \\
$\begin{array}{l}\text { Modos de } \\
\text { barrido }\end{array}$ & $\begin{array}{l}\text { Internos del MEB y } \\
\text { externo del sistema } \\
\text { microanalítico }\end{array}$ \\
\hline
\end{tabular}

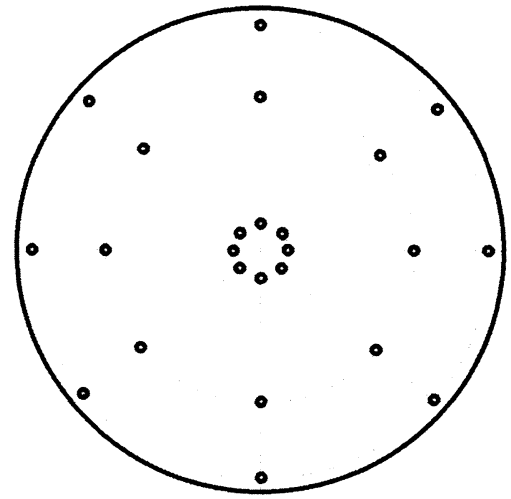

Fig. 1.- Selección de áreas de análisis en un redondo de radio $\mathrm{R}$.

FIG. 1.-Areas selected for analysis in a rod of radius $R$.

$\mathrm{R} / 40$ desde el centro geométrico del redondo y las zonas intermedias en la posición $R / 2$. A partir de las imágenes obtenidas en cada una de las zonas se determina el porcentaje del área total ocupada por las inclusiones (11 y 12).

El sistema de microanálisis EDX, KEVEX 8005 , se calibró según un procedimiento desarrollado en un trabajo previo (13). Las condiciones de análisis y los patrones utilizados se recogen en la tabla II.

TABLA II.- Condiciones de microanálisis cuantitativo EDX TABLE II.- EDX quantitative microanalysis
conditions

\begin{tabular}{|c|c|}
\hline Tipo de detector & $\begin{array}{l}\text { Si (Li), ventana } \\
\text { de } \mathrm{Be}(8 \mu \mathrm{m})\end{array}$ \\
\hline $\begin{array}{l}\text { Potencial de } \\
\text { aceleración }\end{array}$ & $15 \mathrm{KeV}$ \\
\hline Distancia de trabajo & $35 \mathrm{~mm}$ \\
\hline Patrones de calibración & $\begin{array}{l}\text { Para } \mathrm{S} \text { se empleó } \\
\text { pirita }\left(\mathrm{FeS}_{2}\right) ; \\
\text { para } \mathrm{Cr}, \mathrm{Mn}, \mathrm{Fe} \text { y Ni, } \\
\text { metales puros }\end{array}$ \\
\hline $\begin{array}{l}\text { Número de inclu- } \\
\text { siones de sulfuro } \\
\text { analizadas } \\
\text { en cada material }\end{array}$ & 24 a 30 \\
\hline $\begin{array}{l}\text { Tamaño de las } \\
\text { inclusiones analizadas }\end{array}$ & $\begin{array}{l}\text { De } 1,5 \mu \mathrm{m} \text { a } 5 \mu \mathrm{m} \\
\text { (el límite superior } \\
\text { depende de las } \\
\text { inclusiones presentes } \\
\text { en el material }\end{array}$ \\
\hline
\end{tabular}

Rev. Metal. Madrid, 35 (3), 1999 
Para determinar la composición química de los sulfuros se seleccionaron inclusiones distribuidas por toda la sección transversal. En la selección se tuvo en cuenta que las áreas exterior, centro y núcleo (Fig. 1) estuvieran homogéneamente representadas. Los patrones de calibración y las muestras se analizaron exactamente en las mismas condiciones experimentales. Considerando que los sulfuros son básicamente MnS y su densidad 4,03 $\mathrm{g} \cdot \mathrm{cm}^{-3}(7)$, el tamaño de las inclusiones que se analicen debería ser superior a $1,80 \mu \mathrm{m}$, rango de Kanaya-Okayama (14); en cualquier caso deben tener un diámetro no inferior a $1,45 \mu \mathrm{m}$, rango de Anderson-Wasler (15) para evitar que existan interferencias de la matriz del acero. Si durante el análisis de alguna de las inclusiones se observa la presencia de señales de níquel (que no se encuentra en los sulfuros y, por lo tanto, corresponde a la matriz), ese análisis se rechaza y se selecciona una nueva inclusión.

\section{RESULTADOS Y DISCUSIÓN}

La tabla III presenta las composiciones químicas de los materiales en estudio, el diámetro de los redondos, así como los valores experimentales de ocupación en volumen de las inclusiones tipo sulfuros, $\mathrm{dA}_{1}$.

Se observa la práctica invariabilidad de las composiciones químicas, de modo que puede decirse que la población estudiada se corresponde con redondos, de diámetros entre 6 y $10 \mathrm{~mm}$, de inoxidables tipo AISI 303 con una composición química típica (\% masa):

\begin{tabular}{cccccccccc}
$\mathrm{Cr}$ & $\mathrm{Ni}$ & $\mathrm{Mn}$ & $\mathrm{C}$ & $\mathrm{Si}$ & $\mathrm{P}$ & $\mathrm{Mo}$ & $\mathrm{Cu}$ & $\mathrm{Co}$ & $\mathrm{N}$ \\
\hline 17,3 & 8,7 & 1,8 & 0,06 & 0,4 & 0,03 & 0,3 & 0,2 & 0,15 & 0,045
\end{tabular}

De cara al estudio, el azufre es el elemento variable, con valores máximo y mínimo de 0,36 y $0,25 \%$, representando bien los típicos valores extremos de fabricación de estos aceros de fácil mecanización.

La tabla IV presenta las composiciones químicas de los sulfuros, a partir de los análisis por espectrometría de dispersión de energías de rayos $\mathrm{X}$ (EDX), acoplado al microscopio electrónico de barrido (MEB). Cada uno de los resultados de la tabla corresponde al promedio de entre 24 y 30 inclusiones individuales en las que no se aprecia interferencia de matriz y que se encuentran distribuidas homogéneamente en las áreas marcadas en la figura 1.

No se encuentran diferencias en la composición química que puedan asociarse al tamaño de la inclusión o su posición relativa (Fig. 1). Por tanto, en la tabla se incluye únicamente el promedio y la desviación estándar de la medida, entre paréntesis. Las composiciones químicas de los sulfuros de los distintos redondos muestran diferencias estadísticamente significativas (hasta cuatro grupos diferenciales) si se tienen en cuenta los datos de los cuatro elementos en cuestión: azufre, cromo, hierro y manganeso. La tabla IV recoge, además, una serie de parámetros derivados de los datos anteriores. El primero, $\left(\mathrm{dA}_{1}: \mathrm{S}\right)$, expresa la relación existente entre el volumen de sulfuros y el contenido de azufre en el acero.

TABLA III.- Composición química, diámetro de los redondos y contenido de sulfuros de los aceros

TABLE III.-Chemical composition, rod diameters and sulphide contents of the steels

\begin{tabular}{|c|c|c|c|c|c|c|c|c|c|c|c|c|c|c|}
\hline & \multicolumn{12}{|c|}{$\%$ en masa } & \multirow{2}{*}{$\frac{\mathbf{m m}}{\varnothing}$} & \multirow{2}{*}{\begin{tabular}{|r|}
$\% \mathrm{Vol}$ \\
$\mathbf{d A}_{1}$
\end{tabular}} \\
\hline & C & $\mathbf{S i}$ & Mn & $\mathrm{Cr}$ & $\mathbf{N i}$ & Mo & $\mathbf{P}$ & $\mathbf{S}$ & $\mathrm{Cu}$ & Co & $\mathbf{N}$ & 0 & & \\
\hline BJ52 & 0,064 & 0,48 & 1,75 & 17,17 & 8,67 & 0,28 & 0,040 & 0,362 & 0,16 & 0,10 & 0,054 & 0,0145 & 10,0 & 2,11 \\
\hline BJ45 & 0,064 & 0,36 & 1,80 & 17,32 & 8,69 & 0,30 & 0,030 & 0,277 & 0,21 & 0,11 & 0,051 & 0,0094 & 10,0 & 1,56 \\
\hline BC78 & 0,055 & 0,41 & 1,91 & 17,21 & 8,61 & 0,38 & 0,027 & 0,296 & 0,29 & 0,29 & 0,042 & 0,0096 & 7,9 & 1,67 \\
\hline BC75 & 0,051 & 0,35 & 1,89 & 17,34 & 8,72 & 0,31 & 0,028 & 0,349 & 0,20 & 0,17 & 0,049 & 0,0099 & 7,9 & 1,87 \\
\hline AT45 & 0,054 & 0,37 & 1,79 & 17,38 & 8,72 & 0,38 & 0,033 & 0,246 & 0,26 & 0,16 & 0,046 & 0,0097 & 6,5 & 1,38 \\
\hline BT13 & 0,060 & 0,37 & 1,83 & 17,23 & 8,59 & 0,31 & 0,029 & 0,303 & 0,20 & 0,12 & 0,039 & 0,0071 & 6,3 & 1,90 \\
\hline AT00 & 0,055 & 0,44 & 1,89 & 17,29 & 8,69 & 0,35 & 0,033 & 0,273 & 0,25 & 0,16 & 0,044 & 0,0131 & 6,0 & 1,71 \\
\hline AT47 & 0,056 & 0,54 & 1,88 & 17,27 & 8,61 & 0,33 & 0,033 & 0,245 & 0,24 & 0,16 & 0,048 & 0,0086 & 6,0 & 1,60 \\
\hline AT46 & 0,059 & 0,44 & 1,66 & 17,22 & 8,67 & 0,36 & 0,037 & 0,331 & 0,29 & 0,18 & 0,040 & 0,0141 & 6,0 & 2,05 \\
\hline AT56 & 0,054 & 0,35 & 1,79 & 17,30 & 8,71 & 0,36 & 0,036 & 0,310 & 0,24 & 0,15 & 0,050 & 0,0091 & 6,0 & 1,80 \\
\hline$\overline{\mathrm{X}}$ & 0,057 & 0,41 & 1,82 & 17,27 & 8,67 & 0,34 & 0,033 & 0,299 & 0,23 & 0,16 & 0,046 & 0,0102 & & \\
\hline$\sigma_{n-1}$ & 0,004 & 0,06 & 0,08 & 0,07 & 0,05 & 0,04 & 0,004 & 0,040 & 0,04 & 0,05 & 0,005 & 0,0021 & & \\
\hline
\end{tabular}


Los valores de $\left(\mathrm{dA}_{1}: \mathrm{S}\right)$ varían entre 5,37 y 6,53, a pesar de que, si se asumiera la práctica insolubilidad del azufre en el acero y que las composiciones químicas y las densidades de los sulfuros fuesen invariables, debería de cumplirse que

$$
\mathrm{dA}_{1}: \mathrm{S}=\mathrm{K}
$$

Pero también se observa en la tabla IV que existen diferencias apreciables en las composiciones químicas de los sulfuros de los distintos aceros. Una forma de expresar estas diferencias de composiciones químicas de los sulfuros es el parámetro $\left[(\Sigma \mathrm{Me})_{\mathrm{at}}:(\mathrm{S})_{\mathrm{at}}\right]$, que relaciona la suma de átomos metálicos del sulfuro con los átomos de azufre del propio sulfuro. Por 10 tanto, el parámetro expresa la mayor o menor participación metálica en el sulfuro o también su mayor o menor riqueza en azufre. Así pues, para $\left[(\Sigma \mathrm{Me})_{\mathrm{at}}:(\mathrm{S})_{\mathrm{at}}\right]=1$ tendríamos la fórmula MeS, mientras que para una estequiometría $\mathrm{Mn}_{1,0} \mathrm{Cr}_{0,15} \mathrm{Fe}_{0,05} \mathrm{~S}_{0,8}$ (es decir $\mathrm{Me}_{1,2} \mathrm{~S}_{0,8}$ ) tendríamos un valor $\left[(\Sigma \mathrm{Me})_{\mathrm{at}}:(\mathrm{S})_{\mathrm{at}}\right]=1,2: 0,8=1,5$. Así pues podría decirse que las composiciones quí-

TABLA IV.- Composición química de los sulfuros: Parámetros calculados a partir de los datos anteriores

TABLE IV.-Chemical composition of the sulphides: Calculated parameters from previous data

\begin{tabular}{|c|c|c|c|c|c|c|c|c|}
\hline & \multicolumn{4}{|c|}{ Sulfuros } & & & & \\
\hline & \multicolumn{4}{|c|}{$\%$ átomos $\left(\sigma_{n-1}\right)$} & \multirow{2}{*}{$\frac{(1)}{\mathrm{dA}_{1}: \mathrm{S}}$} & \multirow{2}{*}{$\frac{(2)}{(\Sigma \mathrm{Me})_{\mathrm{at}} \cdot(\mathrm{S})_{\mathrm{at}}}$} & \multirow{2}{*}{$\frac{(3)}{(\mathrm{Mn})_{\mathrm{m}}:(\mathrm{S})_{\mathrm{m}}}$} & \multirow{2}{*}{$\frac{(4)}{\operatorname{Calc}\left[\mathrm{dA}_{1}: \mathrm{S}\right]}$} \\
\hline & $S$ & $\mathrm{Cr}$ & $\mathrm{Mn}$ & $\mathrm{Fe}$ & & & & \\
\hline BJ52 & $\begin{array}{l}47,03 \\
(2,26)\end{array}$ & $\begin{array}{c}5,42 \\
(0,78)\end{array}$ & $\begin{array}{l}44,45 \\
(1,34)\end{array}$ & $\begin{array}{c}3,10 \\
(1,30)\end{array}$ & 5,83 & 1,13 & 2,82 & 5,76 \\
\hline BJ45 & $\begin{array}{l}46,37 \\
(2,55)\end{array}$ & $\begin{array}{c}5,06 \\
(0,75)\end{array}$ & $\begin{array}{l}45,50 \\
(1,57)\end{array}$ & $\begin{array}{c}3,07 \\
(1,55)\end{array}$ & 5,63 & 1,16 & 3,79 & 5,92 \\
\hline $\mathrm{BC} 78$ & $\begin{array}{l}48,15 \\
(2,18)\end{array}$ & $\begin{array}{c}5,49 \\
(0,71)\end{array}$ & $\begin{array}{l}42,24 \\
(0,90)\end{array}$ & $\begin{array}{l}4,12 \\
(1,59)\end{array}$ & 5,64 & 1,08 & 3,77 & 5,50 \\
\hline BC75 & $\begin{array}{l}48,16 \\
(1,62)\end{array}$ & $\begin{array}{c}5,12 \\
(0,71)\end{array}$ & $\begin{array}{l}44,48 \\
(0,86)\end{array}$ & $\begin{array}{c}2,24 \\
(1,05)\end{array}$ & 5,37 & 1,08 & 3,16 & 5,50 \\
\hline AT45 & $\begin{array}{l}47,84 \\
(2,66)\end{array}$ & $\begin{array}{c}5,76 \\
(0,95)\end{array}$ & $\begin{array}{l}42,11 \\
(1,15)\end{array}$ & $\begin{array}{c}4,29 \\
(2,35)\end{array}$ & 5,62 & 1,09 & 4,25 & 5,55 \\
\hline BT13 & $\begin{array}{l}45,04 \\
(2,05)\end{array}$ & $\begin{array}{c}5,86 \\
(0,62)\end{array}$ & $\begin{array}{l}45,27 \\
(1,00)\end{array}$ & $\begin{array}{c}3,83 \\
(1,44)\end{array}$ & 6,27 & 1,22 & 3,52 & 6,24 \\
\hline AT00 & $\begin{array}{l}45,53 \\
(1,81)\end{array}$ & $\begin{array}{c}5,25 \\
(0,67)\end{array}$ & $\begin{array}{l}45,61 \\
(0,75)\end{array}$ & $\begin{array}{c}3,61 \\
(1,36)\end{array}$ & 6,28 & 1,20 & 4,04 & 6,13 \\
\hline AT47 & $\begin{array}{l}46,00 \\
(1,47)\end{array}$ & $\begin{array}{c}5,72 \\
(0,58)\end{array}$ & $\begin{array}{l}44,06 \\
(1,08)\end{array}$ & $\begin{array}{c}4,22 \\
(1,43)\end{array}$ & $(6,53)$ & $(1,17)$ & 4,48 & $(5,97)$ \\
\hline AT46 & $\begin{array}{l}45,87 \\
(1,69)\end{array}$ & $\begin{array}{c}6,37 \\
(0,67)\end{array}$ & $\begin{array}{l}43,82 \\
(1,43)\end{array}$ & $\begin{array}{c}3,94 \\
(1,63)\end{array}$ & 6,20 & 1,18 & 2,93 & 6,03 \\
\hline AT56 & $\begin{array}{l}45,91 \\
(1,88)\end{array}$ & $\begin{array}{c}5,84 \\
(0,71)\end{array}$ & $\begin{array}{l}43,54 \\
(1,22)\end{array}$ & $\begin{array}{c}4,71 \\
(1,62)\end{array}$ & 5,82 & 1,18 & 3,37 & 6,03 \\
\hline$\overline{\mathrm{x}}$ & 46,59 & 5,59 & 44,11 & 3,71 & 5,92 & 1,15 & & \\
\hline$\sigma_{n-1}$ & 1,13 & 0,40 & 1,23 & 0,73 & 0,38 & 0,05 & & \\
\hline
\end{tabular}

(1) $\mathrm{dA}_{1}: \mathrm{S}$

$\mathrm{dA}_{1}$ en \% vol ocupado por sulfuros $\mathrm{S}$ en $\%$ masa de $\mathrm{S}$ en el acero

(3) $(\mathrm{Mn})_{\mathrm{m}}:(\mathrm{S})_{\mathrm{m}}$

$(\mathrm{Mn})_{\mathrm{m}}=(\%$ masa $\mathrm{Mn}$ en el acero): 54,94

$(\mathrm{S})_{\mathrm{m}}=(\%$ masa $\mathrm{S}$ en el acero $): 32,06$
(2) $(\Sigma \mathrm{Me})_{a t}:(\mathrm{S})_{3 t}$

$(\Sigma \mathrm{Me})_{\mathrm{at}}=\%$ átomos de $(\mathrm{Cr}+\mathrm{Mn}+\mathrm{Fe})$ en el sulfuro $(\mathrm{S})_{\mathrm{at}}=\%$ átomos de $\mathrm{S}$ en el sulfuro.

(4) Calc $\left[\mathrm{dA}_{1}: \mathrm{S}\right]=-0,17+5,25\left[(\Sigma \mathrm{Me})_{\mathrm{at}}:(\mathrm{S})_{\mathrm{at}}\right]$ 


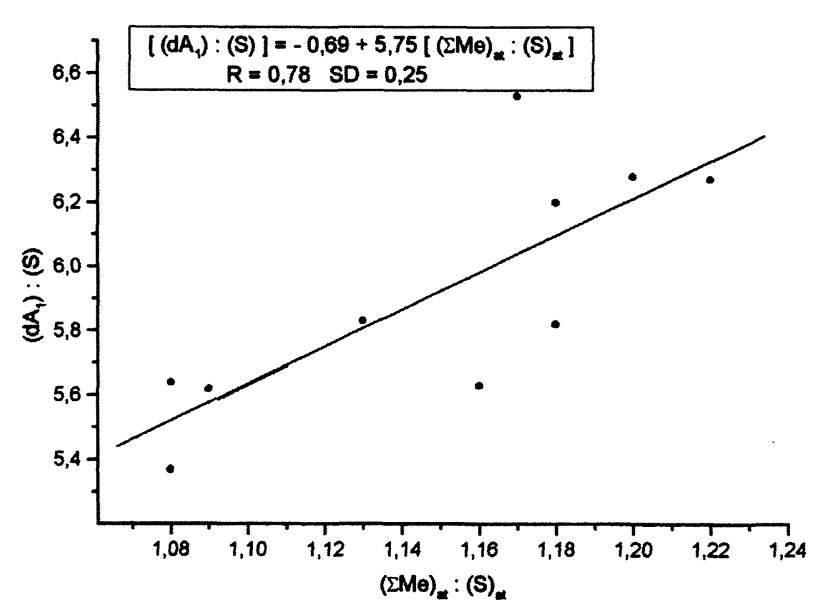

FIG. 2.- Influencia de la composición química de los sulfuros en la relación sulfuros-azufre.

FIG. 2.-Influence of the sulphide chemical composition on the sulphide-sulphur relation.

micas de los sulfuros en los distintos aceros de este estudio son relativamente variables, con valores del parámetro $\left[(\Sigma \mathrm{Me})_{\mathrm{at}}:(\mathrm{S})_{\mathrm{at}}\right]$ comprendidos entre 1,22 y 1,08 .

Cabe plantearse las causas de esta variabilidad de las composiciones químicas de los sulfuros en aceros inoxidables cuyas composiciones químicas son prácticamente invariables. Se ha ensayado una posible relación estadística entre el parámetro $\left[(\mathrm{SMe})_{\mathrm{at}}:(\mathrm{S})_{\mathrm{at}}\right]$ y los niveles de azufre en el acero, sin resultado positivo. Lo mismo ha ocurrido al intentar una correlación empleando en vez del azufre del acero, el parámetro $\left[(\mathrm{Mn})_{\mathrm{at}}:(\mathrm{S})_{\mathrm{at}}\right]$, que tiene en cuenta la relación entre manganeso y azufre en cada acero (Tabla IV). Por lo tanto, cabría pensar que, en la variabilidad de las composiciones químicas de los sulfuros pudieran estar influyendo factores no tenidos en cuenta, como por ejemplo son las condiciones de fabricación en la acería (temperaturas de formación de los sulfuros, condiciones de solidificación, etc.) y las diferentes transformaciones termomecánicas que pueden haber afectado a los equilibrios difusionales acero-sulfuro y a las reorganizaciones cristalinas en los sulfuros.

Asumida pues la variabilidad en las composiciones químicas de los sulfuros y aun en el supuesto de que las estructuras cristalinas y las densidades de los mismos fueran invariables, es necesario admitir en buena lógica que los valores del parámetro $\left(\mathrm{dA}_{1}: \mathrm{S}\right)$ deben depender de las composiciones químicas de los sulfuros. La figura 2 pone de manifiesto esta dependencia.

La figura 3 se obtiene por eliminación del valor $(\Sigma \mathrm{Me})_{\mathrm{at}}:(\mathrm{S})_{\mathrm{at}}=1,17$, ya que mediante la aplicación de la ecuación:

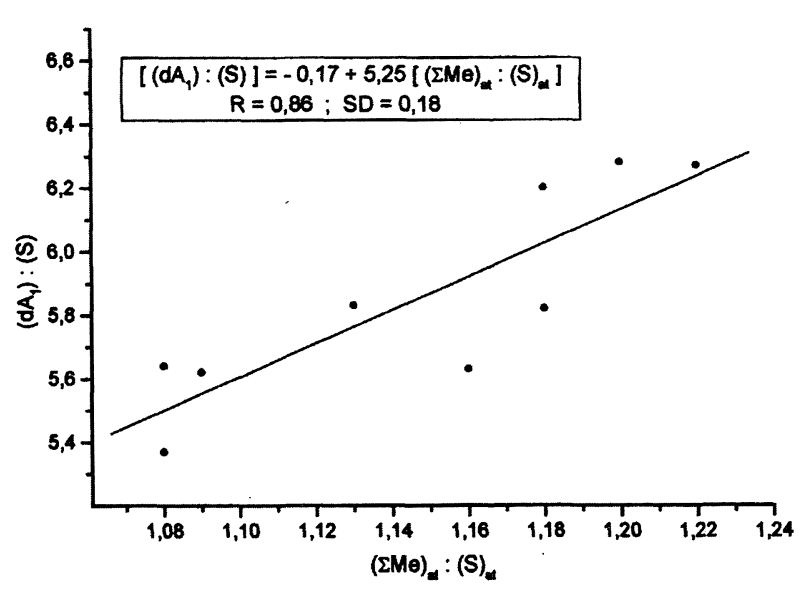

FIG. 3.- Igual que figura 2 (corregida estadísticamente).

FIG. 3.- The same as figure 2 (statistically corrected).

$$
\mathrm{dA}_{1}: \mathrm{S}=-0,69+5,75\left[(\Sigma \mathrm{Me})_{\mathrm{at}}:(\mathrm{S})_{\mathrm{at}}\right]
$$

se obtendría para $\mathrm{dA}_{1}: \mathrm{S}$ el valor 6,04 mientras que el valor medido ha sido 6,53. El criterio de rechazo consiste en eliminar los datos cuyas diferencias entre los valores medidos y los calculados sean iguales o superiores a 1,96 veces la desviación típica.

La nueva correlación es:

$$
\mathrm{dA}_{1}: \mathrm{S}=-0,17+5,25\left[(\Sigma \mathrm{Me})_{\mathrm{at}}:(\mathrm{S})_{\mathrm{at}}\right]
$$

Evidentemente, para un mismo nivel de azufre en el acero se formará mayor cantidad de sulfuros cuanto menor sea la participación del azufre en los mismos (es decir, cuanto mayor sea la participación de los metales en la estequiometría).

La ecuación [3] aplicada al sulfuro clásico MnS en el que $(\Sigma \mathrm{Me})_{\mathrm{at}}=1$ y $(\mathrm{S})_{\mathrm{at}}=1$, daría un valor $\mathrm{dA}_{1}: \mathrm{S}=-0,17+5,25=5,08$.

Para esta misma estequiometría MnS puede establecerse un cálculo teórico de la relación $\mathrm{dA}_{1}: \mathrm{S}$, cálculo basado en el de los volúmenes ocupados por los sulfuros, conocida la masa molecular y las densidades del sulfuro y del propio acero. La tabla $\mathrm{V}$ sistematiza y presenta los resultados de dichos cálculos:

Este valor teórico de 5,28 está bastante próximo al de 5,08 obtenido aplicando la ecuación experimental [2] pero ambos valores están algo alejados de los valores $\left(\mathrm{dA}_{1}: \mathrm{S}\right)$ experimentales individuales de la tabla IV (mínimo valor 5,37; máximo 6,53), lo cual es lógico si se parte de una estequiometría $\mathrm{MnS}$ con importantes diferencias con las estequiometrías reales de los sulfuros analizados en los distintos aceros. 
TABLA V.- Cálculos teóricos de los volúmenes de MnS formados a partir de los contenidos de azufre en el acero TABLE V.-Theoretical calculations of the MnS volumes formed from the sulphur contents in the steel

\begin{tabular}{|c|c|c|c|c|c|c|c|}
\hline 1 & 2 & 3 & 4 & 5 & 6 & 7 & \\
\hline$\%$ masa S & $\%$ masa MnS & $\mathrm{V}_{\mathrm{MnS}}$ & $\%$ masa (acero $)$ & $\mathrm{V}_{\text {acero }}$ & $\mathrm{V}_{\text {total }}$ & $\mathrm{dA}_{1}=\%_{\mathrm{MnS}}$ & $\left(\mathrm{dA}_{1}: \mathrm{S}\right)$ \\
0,25 & 0,678 & 0,168 & 99,322 & 12,575 & 12,740 & 1,319 & 5,28 \\
0,30 & 0,814 & 0,202 & 99,186 & 12,555 & 12,757 & 1,583 & 5,28 \\
0,35 & 0,950 & 0,236 & 99,030 & 12,535 & 12,771 & 1,848 & 5,28 \\
\hline
\end{tabular}

1. Contenido de azufre del acero (tres ejemplos dentro del rango en estudio)

2. $\% \mathrm{MnS}=\% \mathrm{~S} \times[(54,94+32,06): 32,06] ; \% \mathrm{MnS}=\% \mathrm{~S} \times 2,712$

3. $\mathrm{V}_{\mathrm{MnS}}=$ volumen ocupado por la masa de $\mathrm{MnS} ; \mathrm{V}_{\mathrm{MnS}}=\% \mathrm{MnS}: 4,03 *$

4. $\%$ (acero $)=100-\% \mathrm{MnS}$

5. $\mathrm{V}_{\text {acero }}=$ volumen ocupado por $\%$ (acero) $; \mathrm{V}_{\text {acero }}=\%$ (acero) $: 7,9 * *$

6. $\mathrm{V}_{\text {total }}=\mathrm{V}_{\mathrm{MnS}}+\mathrm{V}_{\text {acero }}$

7. $\mathrm{dA}_{1}=\% \mathrm{~V}_{\mathrm{MnS}}=\mathrm{V}_{\mathrm{MnS}} \times 100: \mathrm{V}_{\text {total }}$

* 4,03 $\mathrm{gcm}^{-3}$ (densidad del MnS)

** $7,9 \mathrm{gcm}^{-3}$ (densidad del AISI 303)

Podría obtenerse una mejor aproximación si se parte de lo que en la tabla IV aparece como composición química promedio de los sulfuros de todos los aceros estudiados:

$$
\begin{array}{lll}
\text { (\% en átomos) } & \mathrm{S}=46,59 & \mathrm{Mn}=44,11 \\
& \mathrm{Cr}=5,59 & \mathrm{Fe}=3,71
\end{array}
$$

A esta composición le corresponde la estequiometría aproximada $\mathrm{Mn}_{0,88} \mathrm{Cr}_{0,11} \mathrm{Fe}_{0,07} \mathrm{~S}_{0,93}$, para la que las relaciones de masas $\left[\left(\mathrm{Mn}_{0,88} \mathrm{Cr}_{0,11} \mathrm{Fe}_{0,07} \mathrm{~S}_{0,93}\right):\left(\mathrm{S}_{0,93}\right)\right]$ es de $(87,80$ : $29,82)=2,94$, valor evidentemente distinto de 2,71 que fue el empleado en la tabla $\mathrm{V}(\% \mathrm{MnS}=$ $\% \mathrm{~S} \times 2,712$ ).

Para este valor de 2,94 deducido de la composición química promedio de los sulfuros, la tabla VI - presenta la sistematización de los cálculos y los resultados obtenidos de $\left(\mathrm{dA}_{1}: \mathrm{S}\right)$.

A su vez, aplicando la ecuación [3] a esta misma composición química promedio:

$$
\mathrm{dA}_{1}: \mathrm{S}=-0,17+5,25(1,06: 0,93)=5,81
$$

Una forma de intentar relativizar, en la población estudiada, el efecto de la variabilidad de la composición química de los sulfuros en la relación $\mathrm{dA}_{1}: \mathrm{S}$ consiste en establecer la relación lineal entre los valores de $\mathrm{dA}_{1}$ y $\mathrm{S}$ para encontrar un coeficiente de mínimo error que los relacione.
La figura 4 presenta la recta de regresión lineal, con la ecuación:

$$
\% \mathrm{dA}_{1}=5,88 \% \mathrm{~S}
$$

Ahora resulta evidente que el valor 5,88 está muy cercano a los obtenidos anteriormente, como era de esperar al comparar $\mathrm{dA}_{1}: \mathrm{S}=5,88$ (deducido a partir de datos experimentales) y 5,71 ó 5,81 obtenidos teóricamente pero a partir de una composición química promedio de las composiciones químicas reales de los sulfuros en los diferentes aceros.

\section{RESUMEN Y CONCLUSIONES}

Se ha estudiado una población de 10 redondos de inoxidable AISI 303 (diámetros entre 6 y 10 $\mathrm{mm}$ ), con composiciones químicas típicas $17,3 \%$ $\mathrm{Cr}, 8,7 \% \mathrm{Ni}, 1,8 \% \mathrm{Mn}$, en los que el azufre era variable entre 0,25 y $0,36 \%$ (masa), determinando mediante análisis metalográfico de imágenes el porcentaje de ocupación en volumen de los sulfuros, $\left(\mathrm{dA}_{1}\right)$, así como las composiciones químicas de los mismos utilizando EDX acoplado al MEB. Mediante estos procedimientos se han establecido los valores experimentales $\left(\mathrm{dA}_{1}: \mathrm{S}\right)$, comprobándose una amplia variabilidad entre un valor mínimo de 5,37 y otro máximo de 6,53. Dicha variabilidad no es compatible con el valor constante $\left(\mathrm{dA}_{1}: \mathrm{S}\right)=\mathrm{K}$, 
TABLA VI.- Cálculos teóricos de los volúmenes de sulfuros Mn0,88Cr0,11Fe0,07S0,93 formados a partir de los contenidos de $\mathrm{S}$ en el acero

TABLE VI.- Theoretical calculations of the $\mathrm{MnO} 0,88 \mathrm{Cr} 0,11 \mathrm{Fe} 0,07 \mathrm{SO}, 93$ sulphides formed from the $\mathrm{S}$ contents in the steel

\begin{tabular}{|c|c|c|c|c|c|c|c|}
\hline$\%$ masa S & \% masa MeySx & $\mathrm{V}_{\text {MeySx }}$ & \% masa (acero) & $\mathrm{V}_{\text {acero }}$ & $\mathrm{V}_{\text {total }}$ & $\mathrm{dA} 1=\% \mathrm{~V}_{\text {MeySx }}$ & $\left(\mathrm{dA}_{1}: \mathrm{S}\right)$ \\
\hline 0,25 & 0,735 & 0,182 & 99,265 & 12,565 & 12,747 & 1,428 & 5,71 \\
0,30 & 0,882 & 0,219 & 99,118 & 12,547 & 12,766 & 1,715 & 5,72 \\
0,35 & 1,029 & 0,255 & 98,971 & 12,528 & 12,783 & 1,995 & 5,70 \\
\hline
\end{tabular}

deducible de los supuestos de práctica insolubilidad del azufre en el acero y de la invariabilidad de la composición química y la densidad de los sulfuros formados. Sin embargo, a pesar de ser muy parecidas las composiciones químicas de todos los aceros, existen diferencias en las composiciones químicas de los sulfuros y puede establecerse una relación $\mathrm{dA}_{1}: \mathrm{S}=-0,17+5,25\left[(\Sigma \mathrm{Me})_{\mathrm{at}}:(\mathrm{S})_{\mathrm{at}}\right]$ que tiene en cuenta la dependencia de la cantidad de sulfuros que pueden formarse a partir de una cierta cantidad de azufre en el acero, en función de la composición química de dichos sulfuros de modo que, como resulta lógico, cuanto mayor es la participación del azufre en la estequiometría del sulfuro, menor cantidad de sulfuros se formará para un mismo nivel de azufre.

Se deduce, mediante regresión lineal, una relación $\mathrm{dA}_{1}: \mathrm{S}=5,88$ que, aunque relativiza la variabilidad del parámetro $\left(\mathrm{dA}_{1}: \mathrm{S}\right)$ en función de la composición química, puede servir de referencia o término de comparación.

Los cálculos teóricos de $\left(\mathrm{dA}_{1}: \mathrm{S}\right)$ para un sulfuro de estequiometría $\mathrm{MnS}$ dan un valor de 5,28, aleja-

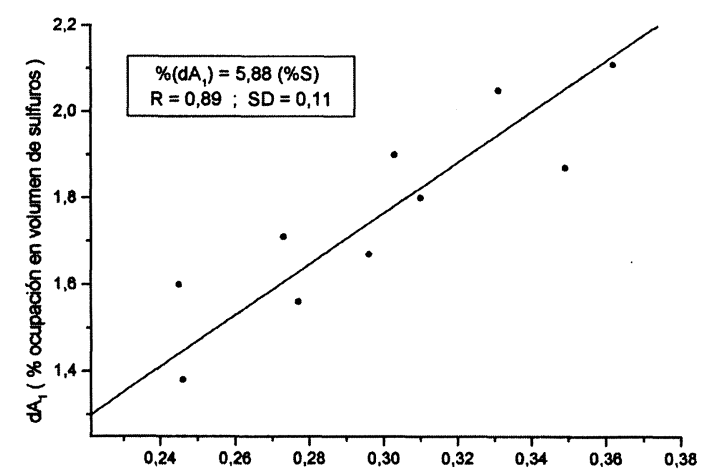

FIG. 4.- Relación entre el contenido de sulfuros y el de azufre en el acero.

FIG. 4.- Relation between sulphide and sulphur contents in the steel. do tanto de los valores individuales como del valor de regresión $(5,88)$. La aproximación, empleando la ecuación empírica [2], da un valor de 5,08 que resulta ser bastante próximo al teórico pero aún más alejado del valor 5,88 . La causa no parece ser otra que la de que las composiciones químicas reales de los sulfuros son distintas de la del MnS teórico.

Para un sulfuro de composición química promedio de las de todos los sulfuros de la población, con fórmula $\mathrm{Mn}_{0,88} \mathrm{Cr}_{0,11} \mathrm{Fe}_{0,07} \mathrm{~S}_{0,93}$, se encuentran valores teóricos $\mathrm{dA}_{1}: \mathrm{S}=5,71$ y valores de 5,81 deducibles de la ecuación empírica [2], ambos ya bastante próximos al de correlación, 5,88.

Indudablemente, se podrían deducir valores más próximos a cada uno de los individuales experimentales mediante cálculos teóricos o aproximaciones, usando la ecuación [2], teniendo en cuenta las composiciones químicas reales de los sulfuros de cada uno de los aceros (Tabla IV).

Parece deducible que la composición química de los sulfuros en el AISI 303 puede ser discretamente variable aun siendo prácticamente invariable la composición química del acero. Ello podría significar que las condiciones de solidificación y las transformaciones termomecánicas pueden influir, dando como resultado variaciones en la composición química de los sulfuros que hacen que no sea estrictamente planteable una relación del tipo $\mathrm{dA}_{1}: \mathrm{S}=\mathrm{K}$. Por lo tanto, si esto es cierto para sulfuros con la misma organización cristalina (y prácticamente la misma densidad), la invalidez de la relación simple $\left(\mathrm{dA}_{1}\right):(\mathrm{S})=\mathrm{K}$ se agudiza en el caso de la formación de sulfuros, en los que no sólo varía la composición química sino, además, la densidad de los mismos.

\section{REFERENCIAS}

1. M. Henthorne. Sulfide inclusions in steel. Materials/Metalworking technology series. ASM N 6. Ohio, 1975: 445-458. 
2. Kovach. Sulfide inclusions in steel, materials/Metalworking technology series ASM N 6. Ohio, 1975: 459-479.

3. Y. Fujiwara, R. Nemoto, K. Osozawa. Proc. Stainless Steels'87. The Institute of Metals. York, 1987: 224-233.

4. A. Paul, J. Botella, J.A. Odriozola. "Determinación experimental de la solubilidad del azufre en el inoxidable $18 \mathrm{Cr} 8 \mathrm{Ni}$ : Relación cuantitativa entre sulfuros de manganeso y azufre en el AISI 304". VI Reunión Nacional de Materiales. S. Sebastián 1999. Aceptado.

5. C.W. Kovach, R.G. Wells, A. Moskowitz. Trans. ASM 61, 1968: 575-581.

6. R. Kiessling. Sulfide inclusions in steel. materials/Metalworking technology series. ASM N6. Ohio, 1975: 104-122.

7. Ficha 6-518, JCPDS-ICDD, 1989.

8. ASTM E 986-86. Standard practice for scanning electron microscope performance characterization. Annual book of ASTM Standards. Vol. 03.01: Metals-Mechanical Testing. Elevated and low temperature test; Metallography. ASTM-Philadelphia 1992.
9. R. Lagneborg, S. Ekelund, T. Werlefors. Combined Image and X-ray analysis in scanning electron microscope for automatic multiparameter characterization of non-metallic inclusions. Swedish Institute for Metals Research. Stockholm (Suecia).

10. J. C. OpPenheIm. Quantitative microscopy and image analysis. D.J. Diaz, Ed. ASM International, Materials Park, Ohio, 1995: 65.

11. J. F. Almagro, M. A. Heredia. Actas de la XVI Reunión bienal de la S.E.M.E., Puerto Real, Cádiz, 1990: 13.

12. R. H. GeIss. Digital image processing. Microanalysis Course, Kevex Corp., 1.988.

13. J. Almagro Bello. Microanálisis cuantitativo mejorado de aceros por EDX-SEM. Informe EUR 16701 ES. Oficina de publicaciones oficiales de las Comunidades Europeas. Serie: Investigación técnica acero - Medidas y análisis. ISBN 92-828-0841-6. Luxemburgo. 1.997.

14. K. Kanaya, S. Okayama. J. Phys. D. Vol 5, 1972:43.

15. C.A. Anderson, M.F. Hasler. Proc. 4th Intl. Conf. On X-ray optics and microanalysis (R. Castaign, P. Deschamps and J. Philibert, eds) Hermann, Paris. 1966:310. 